\title{
The importance of chitobiase and $\mathrm{N}$-acetylglucosamine (GlcNAc) uptake in $N, N^{\prime}$-diacetylchitobiose $\left[(\mathrm{GlcNAc})_{2}\right]$ utilization by Serratia marcescens 2170
}

Correspondence

Takeshi Watanabe

wata@agr.niigata-u.ac.jp

Received 21 December 2007 Revised 21 February 2008 Accepted 22 February 2008

\author{
Tadayuki Toratani, Toshihiro Shoji, Tomonori Ikehara, Kazushi Suzuki \\ and Takeshi Watanabe
}

Department of Applied Biological Chemistry, Faculty of Agriculture, Niigata University, 8050 Ikarashi-2, Niigata 950-2181, Japan

\section{INTRODUCTION}

Chitin is the second most abundant biopolymer in nature and is composed of $\beta$-1,4-linked $N$-acetylglucosamine (GlcNAc) residues (Shahidi et al., 1999). Serratia marcescens 2170 is one of the most effective bacteria for the degradation of chitin. This bacterium is known as an insect pathogen, and chitinase plays an important role in its virulence, together with protease and lecithinase (Grimont \& Grimont, 1978). When S. marcescens is cultivated in the presence of chitin, four chitinases $(\mathrm{A}, \mathrm{B}, \mathrm{C} 1$ and $\mathrm{C} 2$ ) and a $21 \mathrm{kDa}$ chitin-binding-protein (CBP21) can be detected in the culture supernatant (Suzuki et al., 1998). N, $N^{\prime}$ Diacetylchitobiose $\left[(\mathrm{GlcNAc})_{2}\right]$ is the minimal substrate for chitinase and CBP21 induction (Watanabe et al., 1997), and is the major product of chitin hydrolysis by chitinases of this bacterium (Suzuki et al., 2002). Therefore,

Abbreviations: GlcNAc, $N$-acetylglucosamine; (GlcNAc) $)_{2}, N, N^{\prime}$-diacetylchitobiose; 4-MU-GlcNAc, 4-methylumbelliferyl $N$-acetyl- $\beta$-D-glucosaminide; PTS, phosphoenolpyruvate-dependent sugar phosphotransferase system; WS-chitin, water-soluble chitin.
(GlcNAc) $)_{2}$ is considered to be the key molecule in the recognition and utilization of chitin. It has been shown that the uptake of $(\mathrm{GlcNAc})_{2}$ is mediated by the phosphoenolpyruvate-dependent sugar phosphotransferase system (PTS), and a (GlcNAc) $)_{2}$-specific enzyme II permease is involved in the PTS system in S. marcescens 2170 (Uchiyama et al., 2003). The PTS sugars are phosphorylated during translocation across the inner membrane. The generated $(\mathrm{GlcNAc})_{2}-6 \mathrm{P}$ in the cytoplasm is presumably hydrolysed by phosphochitobiase to GlcNAc-6P and GlcNAc. Disruption of the (GlcNAc) $)_{2}$-specific enzyme II permease encoded by the chb operon drastically reduces the production of chitinases and CBP21, and impairs growth on colloidal chitin (Uchiyama et al., 2003).

S. marcescens has a gene encoding a chitobiase of $98.5 \mathrm{kDa}$ and 885 amino acids, including the signal sequence (Tews et al., 1996). Chitobiase hydrolyses the $\beta-1,4$-glycosidic bond in $(\mathrm{GlcNAc})_{2}$ and chito-oligosaccharides. Many chitinolytic bacteria possess $\beta$ - $N$-acetylglucosaminidases, like the chitobiase of $S$. marcescens (Bassler et al., 1991; Tsujibo et al., 2000; Matsuo et al., 1999; Yang et al., 2006). 
It has been reported that $\beta$-N-acetylglucosaminidases located in the periplasm of Vibrio furnissii hydrolyse chito-oligosaccharides to GlcNAc (Bassler et al., 1991). In addition, GlcNAc produced from chito-oligosaccharides is thought to be taken up by GlcNAc permease (Bassler et al., 1991). However, the importance of chitobiase and GlcNAc uptake for the utilization of (GlcNAc) $)_{2}$ and chitooligosaccharides in S. marcescens is poorly understood. In this study, to clarify the utilization mechanism of degradation products from chitin by $S$. marcescens, the contribution of chitobiase and GlcNAc uptake to the utilization of ( $\mathrm{GlcNAc})_{2}$ was examined.

The gene encoding chitobiase was previously designated chb (Tews et al., 1996). However, this name may cause confusion with the chb operon encoding the genes required for PTS-mediated transport and metabolism of (GlcNAc) $)_{2}$. Therefore, the gene name chb for chitobiase is not used in this paper; instead, the chitobiase gene is tentatively named $c t b$.

\section{METHODS}

Bacterial strains, plasmids and culture conditions. S. marcescens 2170 and its mutants were grown at $33{ }^{\circ} \mathrm{C}$ with shaking in a yeast extract-supplemented minimal (YEM) medium containing various carbon sources (Monreal \& Reese, 1969). The yeast extract concentration in this medium was reduced to $0.025 \%$. Escherichia coli strains were grown at 30 or $37{ }^{\circ} \mathrm{C}$ in Luria-Bertani (LB) medium containing appropriate antibiotics. Growth was monitored by measuring $\mathrm{OD}_{660}$ using a mini-photo 518 photometer (Taitec). Antibiotics were used at the following concentrations: ampicillin, $100 \mu \mathrm{g} \mathrm{ml}^{-1}$; chloramphenicol, $50 \mu \mathrm{g} \mathrm{ml}^{-1}$; kanamycin, $50 \mu \mathrm{g} \mathrm{ml}^{-1}$; tetracycline, $25 \mu \mathrm{g} \mathrm{ml}^{-1}$.

Construction of mutants with disrupted ctb and nagE genes. Mutants were constructed by homologous recombination using pirdependent replication plasmid pFS200 (Uchiyama et al., 2003). A $290 \mathrm{bp}$ internal region of the $c t b$ gene and a $400 \mathrm{bp}$ internal region of the nagE gene were amplified by PCR with primers chb1-F (5'-GACGGCAAAGACTGGGTCATCTATTTCCAC-3') and chb1-R (5'-AACGGCGCCACAAACTGATCCAGGTTTTC-3') for the $c t b$ mutant, and nagE-F (5'-AAGGCGCTGTCGTTCTTCAG-3') and nagE-R ( $5^{\prime}$-GACGCGCGCAGTGATAGATG-3') for the nagE mutant, using chromosomal DNA of $S$. marcescens 2170 as a template. PCR products were cloned into pT7Blue T-vector or pUC119 and sequenced. From the plasmids, target regions were excised and cloned into pFS200. The resulting plasmids were first introduced into

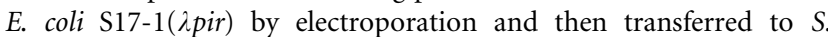
marcescens 2170 by conjugation, as previously described (Uchiyama et al., 2003). Individual transconjugants were selected on LB agar plates containing chloramphenicol and tetracycline. Disruption of the target genes was confirmed by sequencing the PCR product obtained with primers complementary to the sequence within the chloramphenicol-resistance gene and to the sequence outside of the target region.

Intracellular, extracellular and total chitobiase activities. The culture supernatant (extracellular fraction) was prepared by centrifugation at $8000 \mathrm{~g}$ for $5 \mathrm{~min}$. After removing the supernatant, the cell pellet was suspended in $0.1 \mathrm{M}$ sodium phosphate buffer ( $\mathrm{pH}$ 6.0). To prepare the intracellular fraction, the cell suspension was then lysed by sonication for $30-120 \mathrm{~s}$. To measure the total activities containing intra- and extracellular activities, the culture was permeabilized by adding chloroform at a ratio of $100 \mu \mathrm{ml}^{-1}$ and vortexing vigorously for $10 \mathrm{~s}$. Chitobiase activity was measured using 4-methylumbelliferyl $N$-acetyl- $\beta$-D-glucosaminide (4-MU-GlcNAc) (Sigma) as the substrate. A $50 \mu \mathrm{l}$ sample was mixed with $500 \mu \mathrm{l}$ of $10 \mu \mathrm{M} 4-\mathrm{MU}$ GlcNAc in $100 \mathrm{mM}$ sodium phosphate buffer ( $\mathrm{pH}$ 6.0). After incubation for $5 \mathrm{~min}$ at $37{ }^{\circ} \mathrm{C}$, the reaction was stopped by adding $500 \mu \mathrm{l} 0.2 \mathrm{M} \mathrm{Na}_{2} \mathrm{CO}_{3}$. The fluorescence of the liberated 4-MU was measured with excitation at $360 \mathrm{~nm}$ and emission at $450 \mathrm{~nm}$ using an RF-5300 spectrofluorophotometer (Shimadzu). One unit of chitobiase activity was defined as the amount of enzyme that liberates $1 \mathrm{nmol} 4$-MU per min.

SDS-PAGE and activity staining. SDS-PAGE was carried out as described by Laemmli (1970) using $12.5 \%$ polyacrylamide gels under reducing conditions. After electrophoresis, the gel was washed three times with $2.5 \%$ Triton X-100 for $60-90$ min to remove SDS and renature the proteins. The gel was then incubated for $60 \mathrm{~min}$ in $0.1 \mathrm{M}$ sodium phosphate buffer ( $\mathrm{pH}$ 6.0). The polyacrylamide gel was overlaid with an agarose gel containing $0.1 \mathrm{mM} 4-\mathrm{MU}$-GlcNAc in $0.1 \mathrm{M}$ sodium phosphate buffer ( $\mathrm{pH}$ 6.0) for $10 \mathrm{~min}$. Enzyme activity was detected as fluorescent bands under UV light. Proteins separated by SDS-PAGE were stained with Coomassie brilliant blue R-250.

Chemicals. GlcNAc was purchased from Seikagaku Kogyo Co. Water-soluble chitin (WS-chitin) and $N, N^{\prime}$-diacetylchitobiose were kindly provided by Yaizu Suisan Chemical Co. The degree of deacetylation of the WS-chitin is $38.8 \%$, and its approximate molecular mass is $200-300 \mathrm{kDa}$. WS-chitin is soluble in water due to partial deacetylation and the limited size of the chitin chain. Therefore, it is possible to measure cell growth in medium containing WS-chitin by optical density.

\section{RESULTS}

\section{Arrangement of genes and construction of mutants}

$(\mathrm{GlcNAc})_{2}$ is the major product of chitin hydrolysis by chitinases of S. marcescens 2170 (Suzuki et al., 2002). Uptake of $(\mathrm{GlcNAc})_{2}$ via a (GlcNAc) $)_{2}$-specific enzyme II permease has been demonstrated previously (Uchiyama et al., 2003). However, the presence of chitobiase in this organism suggests that $(\mathrm{GlcNAc})_{2}$ is in part hydrolysed by chitobiase to GlcNAc, and then taken up by GlcNAc permease. $S$. marcescens has a nag operon (nagEBACD), highly homologous to the E. coli nag operon, which is involved in GlcNAc uptake and metabolism (Plumbridge, 1989; Postma et al., 1993). The nagE gene encodes GlcNAc-specific enzyme II permease (Lengeler et al., 1994). The nag operon is positioned near the $c t b$ gene on the genome of $S$. marcescens. The $c t b$ gene is clustered with genes homologous to $E$. coli $y b f M$ and $y b f N$, which are genes with unknown function. $n a g E$ and nagBACD are transcribed in opposite directions. To clarify the contribution of chitobiase and GlcNAc uptake to the utilization of chitin, $c t b$ and nagE mutants were constructed. Mutants were constructed by homologous recombination using pFS200, a pir-dependent replication plasmid containing a chloramphenicol-resistance gene. Briefly, the internal regions of the target genes were cloned into pFS200. The resulting plasmids were transferred from $E$. coli S17-1( $\lambda$ pir) to $S$. marcescens 2170 by conjugation, and 
transconjugants were selected based on chloramphenicol resistance. Chloramphenicol-resistant transconjugants should contain the respective plasmids integrated into the target gene on the chromosome by homologous recombination, leading to two incomplete copies of the target gene. Integration of the respective plasmids into the chromosomal DNA of S. marcescens 2170 was confirmed by PCR and the desired mutants were obtained.

\section{Chitobiase production by S. marcescens 2170}

When S. marcescens QMB1466 is cultivated in a medium containing chitin, $(\mathrm{GlcNAc})_{2}$-hydrolysing activity, which is probably due to chitobiase, is detected in the culture supernatant (Monreal \& Reese, 1969). In contrast, chitobiase produced in E. coli carrying the S. marcescens ctb gene is secreted into the periplasm (Tews et al., 1996). Therefore, we first investigated the localization of chitobiase in S. marcescens. Wild-type 2170 and the $c t b$ mutant were grown to mid-exponential phase in YEM medium containing $0.2 \%$ WS-chitin. The $c t b$ mutant grew more slowly than the wild-type strain. Extra- and intracellular fractions were analysed by SDS-PAGE and activity staining with 4-MU-GlcNAc (Fig. 1). A thin protein band of approximately $96 \mathrm{kDa}$, which coincided with the predicted molecular mass of chitobiase, was detected in the extracellular fraction of the wild-type strain in SDSPAGE analysis. This band was considered to be chitobiase because it was not detected in the $c t b$ mutant. In activitystaining analysis, only a single fluorescent band at the position corresponding to the protein band of chitobiase was observed in both fractions of the wild-type strain. This fluorescent band was not observed in the $c t b$ mutant. Accordingly, chitobiase was found to be located both inside and outside the cell. It is worth noting that chitinases and CBP21 were produced normally in the $c t b$ mutant.

We then tried to determine extra- and intracellular chitobiase activity. However, attempts to measure intracellular activity quantitatively failed because chitobiase was inactivated by sonication to disrupt the cells. Therefore, total activity, representing the sum of extra- and intracellular activity, was measured as described in Methods. Significant activities were detected in the wild-type strain, while activities were barely detected in the $c t b$ mutant, indicating that chitobiase is the main enzyme that hydrolyses this substrate. In the wild-type strain, total activity $\left(10.1 \pm 2.2 \mathrm{U} \mathrm{ml}^{-1}\right.$ per $\mathrm{OD}_{660}$ unit $)$ was much higher than extracellular activity $\left(3.5 \pm 0.9 \mathrm{U} \mathrm{ml}^{-1}\right.$ per $\mathrm{OD}_{660}$ unit). When the extracellular fraction was analysed by SDS-PAGE, weak protein bands presumably derived from cellular proteins were detected, suggesting that some, but not all, extracellular chitobiase activity was released from the cells by cell lysis. Chitobiase from $S$. marcescens possesses the signal sequence in its precursor form, and is secreted into the periplasm when expressed in E. coli (Tews et al., 1996). Therefore, intracellular chitobiase is suggested to be mainly located in the periplasm, and is more abundant than extracellular chitobiase. The attempt to extract chitobiase from the periplasm by the osmotic shock procedure was unsuccessful. However, this was not unexpected because it has been reported that the periplasmic proteins of $S$. marcescens are resistant to release by osmotic shock (Cerny \& Teuber, 1972).

\section{Growth of S. marcescens strains on various carbon sources}

$(\mathrm{GlcNAc})_{2}$ is the major product of chitin hydrolysis by chitinases of S. marcescens 2170. Because chitobiase extra intra

(a) $\mathrm{wt} \mathrm{cm}$ wt $\mathrm{cm} \mathrm{M}$

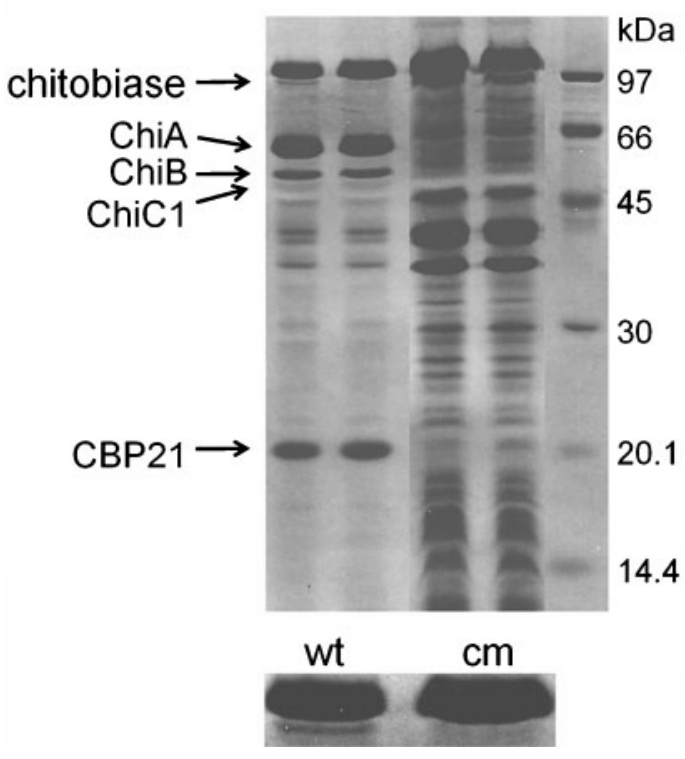

extra intra

(b) wt $\mathrm{cm} \mathrm{wt} \mathrm{cm}$

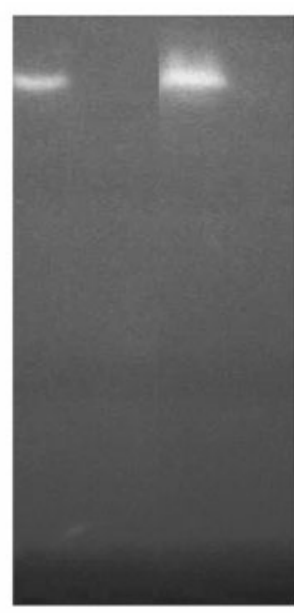

Fig. 1. Chitinase and chitobiase production in S. marcescens 2170 strains. Wild-type 2170 (wt) and ctb mutant (cm) were grown to midexponential phase in YEM medium containing $0.2 \%$ WS-chitin. (a) SDS-PAGE analysis of the extra- and intracellular fractions corresponding to $500 \mu$ culture. $\mathrm{M}$, size marker. The gel portion near $97 \mathrm{kDa}$ extracellular fractions is enlarged below. (b) Activity-staining analysis after SDS-PAGE. Chitobiase activity was detected as fluorescent bands using 4-MUGlcNAc. 
hydrolyses (GlcNAc) $)_{2}$ to GlcNAc, it has been thought to be involved in the utilization of $(\mathrm{GlcNAc})_{2}$. To clarify the involvement of chitobiase in the utilization of (GlcNAc) $)_{2}$, S. marcescens 2170 and its mutants were grown in YEM medium containing $0.1 \%$ glycerol, $0.1 \%$ GlcNAc or $0.1 \%$ $(\text { GlcNAc })_{2}$ (Fig. 2). The wild-type strain grew well on all carbon sources examined. On the other hand, growth of the $c t b$ mutant on $(\mathrm{GlcNAc})_{2}$ was significantly retarded compared with that of the wild-type strain, indicating that chitobiase plays an important role in the utilization of $(\mathrm{GlcNAc})_{2}$.

As described above, chitobiase is suggested to be localized both in the periplasm and outside the cells. When chitobiase hydrolyses $(\mathrm{GlcNAc})_{2}$ in the periplasm and supernatant, GlcNAc permease is predicted to be required partially for the utilization of ( $\mathrm{GlcNAc})_{2}$ but not that of glycerol. To test the above prediction, a mutant for nagE, encoding GlcNAcspecific enzyme II permease, was constructed. The nagE mutant grew very slowly on GlcNAc (Fig. 2b), indicating that NagE is the main permease for GlcNAc. Slow growth of the nagE mutant on GlcNAc suggests slight uptake of GlcNAc through other minor pemeases. On $(\mathrm{GlcNAc})_{2}$, the nagE mutant initially grew slowly, similar to the $c t b$ mutant, and then it showed a greatly reduced growth rate (Fig. 2a). The nagE mutant produced chitobiase on (GlcNAc) $)_{2}$ and total activity at $6 \mathrm{~h}$ cultivation was $15.9 \mathrm{U} \mathrm{ml}^{-1}$ per $\mathrm{OD}_{660}$ unit. The involvement of NagE in the growth on (GlcNAc) suggests that GlcNAc uptake is important for the utilization of $(\mathrm{GlcNAc})_{2}$.

\section{Induction of chitobiase}

Wild-type and mutant strains were grown to mid-exponential phase in YEM medium containing $0.1 \%$ glucose, glycerol, GlcNAc, $(\mathrm{GlcNAc})_{2}$ or glycerol $+(\mathrm{GlcNAc})_{2}$. Total chitobiase activities were measured using 4-MUGlcNAc as substrate (Fig. 3). Chitobiase activity was detected on GlcNAc, $(\mathrm{GlcNAc})_{2}$ and glycerol $+(\mathrm{GlcNAc})_{2}$ in the wild-type strain. Culture on $(\mathrm{GlcNAc})_{2}$ showed fivefold higher activity than that on GlcNAc. Therefore, the expression of chitobiase is induced by GlcNAc and $(\mathrm{GlcNAc})_{2}$, and $(\mathrm{GlcNAc})_{2}$ is a more effective inducer. On the other hand, the $c t b$ mutant did not show significant chitobiase activity on GlcNAc and (GlcNAc) $)_{2}$.

In S. marcescens 2170, the LysR-type transcriptional regulator $\mathrm{ChiR}$ and $(\mathrm{GlcNAc})_{2}$ uptake are essential for the production of all chitinases and CBP21 (Suzuki et al., 2001; Uchiyama et al., 2003). The chbC encodes the (GlcNAc) $2_{2}^{-}$ specific enzyme IIC component of the PTS. Disruption of $c h b C$ drastically reduced the production of chitinases because of a lack of ( $\mathrm{GlcNAc})_{2}$ uptake, as demonstrated previously (Uchiyama et al., 2003). To examine whether $\mathrm{ChiR}$ and $(\mathrm{GlcNAc})_{2}$ uptake is required for the induction of chitobiase, chiR and $c h b C$ mutants were grown in medium containing GlcNAc or (GlcNAc) $)_{2}$. As shown in Fig. 3, the chiR mutant (N1) produced chitobiase normally, indicating that chitobiase is not regulated by ChiR. Since the chbC mutant grew very slowly on (GlcNAc) ${ }_{2}$ (Fig. 2), chitobiase production was compared in medium containing both $0.1 \%$ glycerol and $0.1 \%(\mathrm{GlcNAc})_{2}$, in which the wild-type strain and the $c h b C$ mutant grew equally (Uchiyama et al., 2003). Glycerol does not repress chitobiase production of the wildtype strain, as shown in Fig. 3. On the other hand, chitobiase activity of the $c h b C$ mutant was very low, indicating that uptake of $(\mathrm{GlcNAc})_{2}$ is important for the production of chitobiase. Both mutants produced chitobiase on GlcNAc at the same level as the wild-type strain.

\section{DISCUSSION}

Many chitinolytic bacteria have $\beta$ - $N$-acetylglucosaminidases, which hydrolyse (GlcNAc) ${ }_{2}$ and chito-oligosaccharides to GlcNAc (Bassler et al., 1991; Tsujibo et al., 2000;
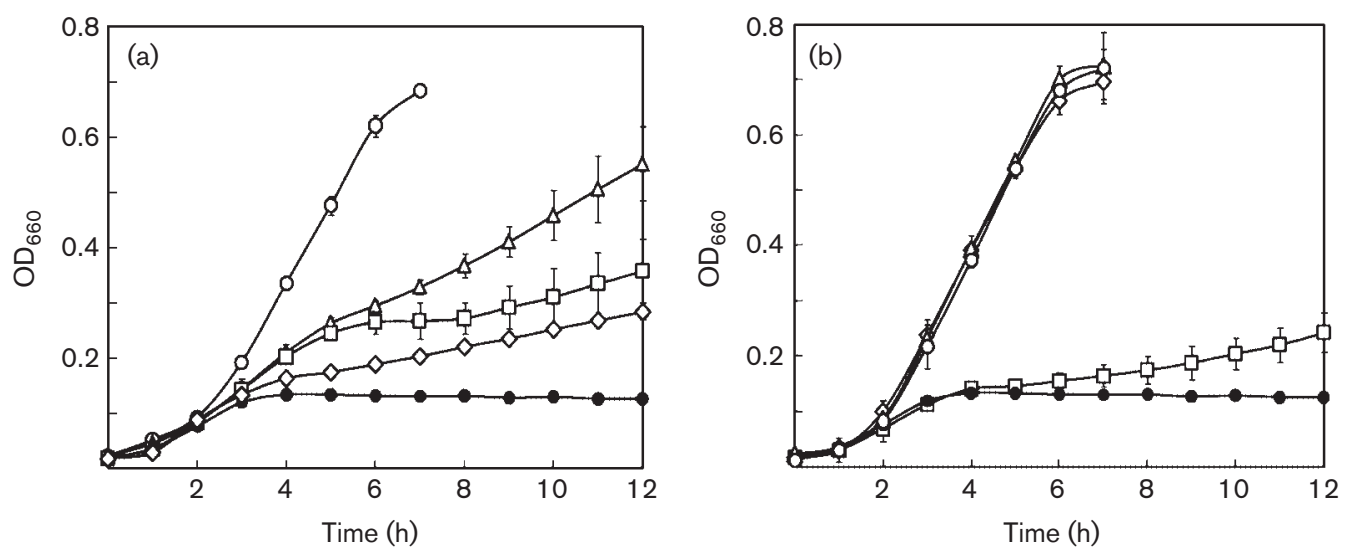

Fig. 2. Growth of $S$. marcescens 2170 strains on YEM medium containing (a) $0.1 \%(\text { GlcNAc) })_{2}$ or (b) $0.1 \%$ GlcNAc. $\bigcirc$, Wildtype; $\triangle$, ctb mutant; $\square$, nagE mutant; $\diamond, c h b C$ mutant; $\bullet$ no carbon source control. Each point represents the mean \pm SD of two to four independent experiments. 


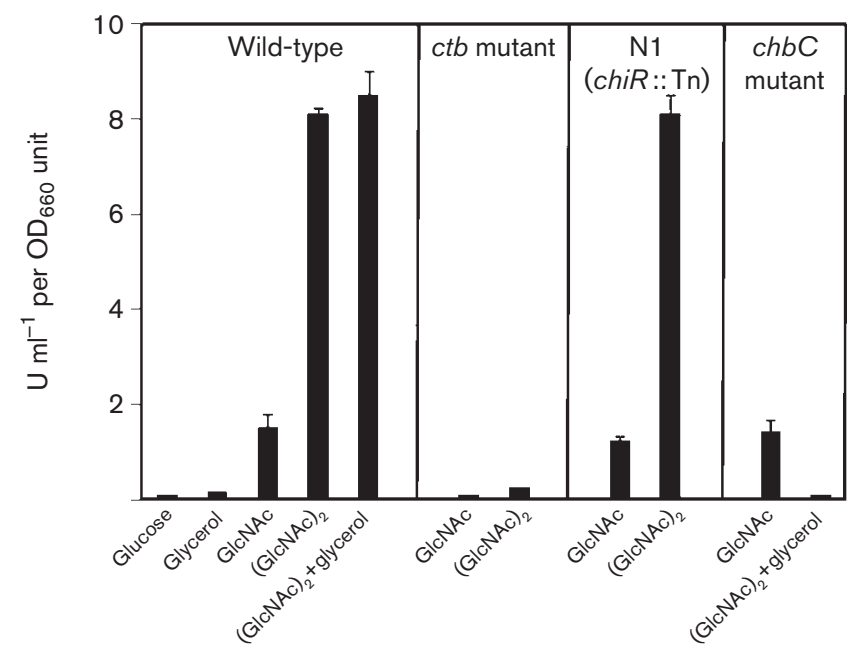

Fig. 3. Total chitobiase activity. Wild-type and mutant strains were grown to mid-exponential phase in YEM medium containing $0.1 \%$ glucose, $0.1 \%$ glycerol, $0.1 \%$ GlcNAc, $0.1 \%$ (GlcNAc) 2 or $0.1 \%$ glycerol $+0.1 \% \quad(\mathrm{GlcNAc})_{2}$. Total chitobiase activities were measured using 4-MU-GIcNAc. Data are means \pm SD of two or three independent experiments.

Matsuo et al., 1999; Yang et al., 2006). This study showed that chitobiase is the main $\beta$-N-acetylglucosaminidase for chitin utilization in $S$. marcescens 2170 , because activity against 4-MU-GlcNAc was hardly detectable in the $c t b$ mutant, and (GlcNAc) $)_{2}$ is the main product of $S$. marcescens chitinases. When S. marcescens 2170 was cultivated on chitin, chitobiase activity was detected both inside and outside the cells. S. marcescens chitobiase possesses a signal sequence in its precursor form and is secreted in the periplasm when cloned in E. coli (Tews et al., 1996). Therefore chitobiase inside the cells of S. marcescens 2170 is considered to be localized in the periplasm.

Uchiyama et al. (2003) showed that (GlcNAc) ${ }_{2}$ molecules are taken up by a (GlcNAc) $)_{2}$-specific permease into the cytoplasm. In addition to uptake as $(\mathrm{GlcNAc})_{2}$, a certain portion of (GlcNAc) $)_{2}$ must be converted to GlcNAc by chitobiase, mainly in the periplasm, and incorporated into the cytoplasm by the GlcNAc-specific enzyme II permease, because $c t b$ and $n a g E$ are both required for normal growth on $(\mathrm{GlcNAc})_{2}$. Therefore, uptake of both $(\mathrm{GlcNAc})_{2}$ and GlcNAc is important for the utilization of (GlcNAc) $)_{2}$ in $S$. marcescens.

Both $c t b$ and nagE mutants are assumed to essentially take up only $(\mathrm{GlcNAc})_{2}$ molecules when they are grown on $(\mathrm{GlcNAc})_{2}$. However, the two mutants grew differently on $(\text { GlcNAc })_{2}$, as shown in Fig. 2(a). The nagE mutant initially grew slowly at a similar rate to the $c t b$ mutant on $(\mathrm{GlcNAc})_{2}$, and then the growth rate was greatly reduced. This observation suggests that most of the (GlcNAc) $)_{2}$ in the periplasm was degraded to GlcNAc by chitobiase when the growth rate was reduced. In the case of $V$. furnissii, mutation of NagE, the sole permease of GlcNAc, resulted in an increase in the generation time from 1.4 to $2.5 \mathrm{~h}$ on $(\text { GlcNAc) })_{2}$ (Bassler et al., 1991). The effect of nagE mutation appeared to be smaller than with S. marcescens. The difference in the effect of the two mutations may be explained by the difference in $(\mathrm{GlcNAc})_{2}$ permeases (ABC transporter in $V$. furnissii; PTS permease in S. marcescens 2170). The affinity for (GlcNAc) $)_{2}$ of the $\mathrm{ABC}$ transporter is much higher than that of the PTS permease (Keyhani et al., 2000). The high-affinity transporter may enable more effective transport under the conditions in which $(\mathrm{GlcNAc})_{2}$ concentration is reduced by the action of $\beta$ $\mathrm{N}$-acetylglucosaminidases.

The $c t b$ mutant, which takes up only (GlcNAc) ${ }_{2}$ molecules on $(\mathrm{GlcNAc})_{2}$, grew more slowly on $(\mathrm{GlcNAc})_{2}$ than on GlcNAc. This means that (GlcNAc) $)_{2}$ is less efficiently fermented by $S$. marcescens 2170 in the absence of chitobiase. Slower fermentation of $(\mathrm{GlcNAc})_{2}$ than GlcNAc was also demonstrated with E. coli (Keyhani \& Roseman, 1997), which belongs to the Enterobacteriaceae, as does S. marcescens. E. coli takes up GlcNAc and $(\mathrm{GlcNAc})_{2}$ by respective PTS permeases (Keyhani \& Roseman, 1997), just like $S$. marcescens, but lacks chitobiase (Yang et al., 2006). Therefore, it is plausible to conclude that $S$. marcescens possesses chitobiase for efficient utilization of $(\mathrm{GlcNAc})_{2}$ by converting it to the more readily fermentable sugar GlcNAc.

Chitobiase was induced by GlcNAc and (GlcNAc) $)_{2}$, and $(\mathrm{GlcNAc})_{2}$ is a more effective inducer. (GlcNAc) $)_{2}$ uptake and ChiR are essential for the induction of chitinases and CBP21 (Uchiyama et al., 2003; Suzuki et al., 2001). The chiR mutant N1 produced chitobiase normally on (GlcNAc) ${ }_{2}$. In contrast, the chbC mutant, defective in the (GlcNAc) $)_{2}$-specific enzyme IIC component of the PTS, did not show any chitobiase activity in a medium containing $(\mathrm{GlcNAc})_{2}$ and glycerol. These results indicate that uptake of (GlcNAc $)_{2}$ is important in the induction of chitobiase as well as chitinases and CBP21. The requirement of (GlcNAc) ${ }_{2}$ uptake for chitobiase production suggests that a certain substrate generated during or after the uptake, such as $(\mathrm{GlcNAc})_{2}-6 \mathrm{P}$, is involved in regulation of the $c t b$ gene. The importance of (GlcNAc) ${ }_{2}$ uptake for chitobiase production also means that the $\operatorname{chbC}$ mutant essentially cannot utilize either GlcNAc or (GlcNAc) $)_{2}$ when supplied with $(\mathrm{GlcNAc})_{2}$ as carbon source, due to the absence of chitobiase. This would explain the very slow growth of the chbC mutant on (GlcNAc) ${ }_{2}$, as shown in Fig. 2. Unlike chitinases and CBP21, chitobiase is not regulated by ChiR. In $S$. marcescens, the $c t b$ gene is clustered with the $y b f M$ and $y b f N$ genes located upstream of $c t b$. Yang et al. (2006) predicted that $y b f M$ is the regulon of $\mathrm{NagC}$, which is a regulator of the nag operon (Vogler \& Lengeler, 1989), because of the presence of a possible NagC-binding site upstream from the $y b f M$ gene in S. marcescens. It has been shown that NagC regulates not only the nag operon, but also the $c h b$ operon, responsible for utilization of $(\mathrm{GlcNAc})_{2}$, and the glmUS genes, involved in the GlcNAc 


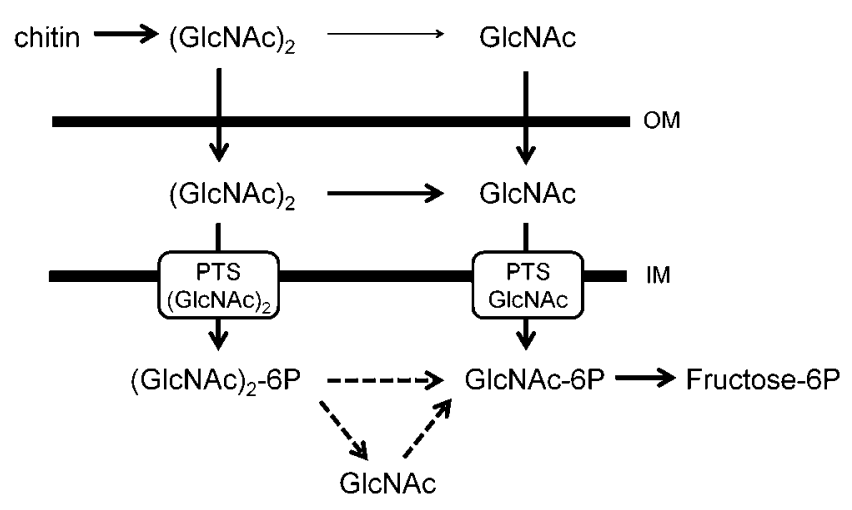

Fig. 4. A model of the catabolic pathway of degradation products from chitin in S. marcescens. Solid arrows indicate the pathways supported by experimental data. Dashed arrows denote the hypothetic pathways with no experimental data. OM, outer membrane; IM, inner membrane.

biosynthetic pathway, in E. coli (Plumbridge \& Pellegrini, 2004; Plumbridge, 1995). The involvement of NagC in chitobiase induction is suggested by the fact that chitobiase is induced by GlcNAc. In addition to $\mathrm{NagC}$, other regulators must be involved in chitobiase induction because chitobiase was more strongly induced by $(\mathrm{GlcNAc})_{2}$ than by GlcNAc.

Fig. 4 illustrates a model of the catabolic pathway of degradation products from chitin in $S$. marcescens deduced from previously obtained results and the present study. $(\mathrm{GlcNAc})_{2}$ is the main product of the action of $S$. marcescens chitinases on chitin. A proportion of $(\mathrm{GlcNAc})_{2}$ molecules enter the periplasm and are taken up into the cytoplasm by the $(\mathrm{GlcNAc})_{2}$-specific PTS permease. The remainder of the $(\mathrm{GlcNAc})_{2}$ is converted to GlcNAc either outside the cells or in the periplasm by chitobiase, which is induced by (GlcNAc) $)_{2}$ uptake. GlcNAc molecules are taken up via the GlcNAc-specific PTS permease. Both $(\mathrm{GlcNAc})_{2}$ and GlcNAc are phosphorylated during translocation across the inner membrane. $(\mathrm{GlcNAc})_{2}-6 \mathrm{P}$ in the cytoplasm is presumably hydrolysed by phosphochitobiase to GlcNAc-6P and GlcNAc, and further catabolized. This model should be confirmed in the near future by uptake studies using radioactively labelled substrates.

\section{REFERENCES}

Bassler, B. L., Yu, C., Lee, Y. C. \& Roseman, S. (1991). Chitin utilization by marine bacteria. Degradation and catabolism of chitin oligosaccharides by Vibrio furnissii. J Biol Chem 266, 24276-24286.

Cerny, G. \& Teuber, M. (1972). Comparative polyacrylamide electrophoresis of periplasmic proteins released from Gram-negative bacteria by polymyxin B. Arch Mikrobiol 82, 361-370.

Grimont, P. A. \& Grimont, F. (1978). The genus Serratia. Annu Rev Microbiol 32, 221-248.

Keyhani, N. O. \& Roseman, S. (1997). Wild-type Escherichia coli grows on the chitin disaccharide, $\mathrm{N}, \mathrm{N}^{\prime}$-diacetylchitobiose, by expressing the cel operon. Proc Natl Acad Sci U $S$ A 94, 14367-14371.

Keyhani, N. O., Wang, L.-X., Lee, Y. C. \& Roseman, S. (2000). The chitin disaccharide, $N, N^{\prime}$-diacetylchitobiose, is catabolized by Escherichia coli and is transported/phosphorylated by the phosphoenolpyruvate:glycose phosphotransferase system. J Biol Chem 275, 33084-33090.

Laemmli, U. K. (1970). Cleavage of structural proteins during the assembly of the head of bacteriophage T4. Nature 227, 680-685.

Lengeler, J. W., Jahreis, K. \& Wehmeier, U. F. (1994). Enzymes II of the phospho enol pyruvate-dependent phosphotransferase systems: their structure and function in carbohydrate transport. Biochim Biophys Acta 1188, 1-28.

Matsuo, Y., Kurita, M., Park, J. K., Tanaka, K., Nakagawa, T., Kawamukai, M. \& Matsuda, H. (1999). Purification, characterization and gene analysis of $\mathrm{N}$-acetylglucosaminidase from Enterobacter sp. G-1. Biosci Biotechnol Biochem 63, 1261-1268.

Monreal, J. \& Reese, E. T. (1969). The chitinase of Serratia marcescens. Can J Microbiol 15, 689-696.

Plumbridge, J. A. (1989). Sequence of the nagBACD operon in Escherichia coli $\mathrm{K} 12$ and pattern of transcription within the nag regulon. Mol Microbiol 3, 505-515.

Plumbridge, J. (1995). Co-ordinated regulation of amino sugar biosynthesis and degradation: the NagC repressor acts as both an activator and a repressor for the transcription of the glmUS operon and requires two separated NagC binding sites. EMBO J 14, 3958-3965.

Plumbridge, J. \& Pellegrini, O. (2004). Expression of the chitobiose operon of Escherichia coli is regulated by three transcription factors: NagC, ChbR and CAP. Mol Microbiol 52, 437-449.

Postma, P. W., Lengeler, J. W. \& Jacobson, G. R. (1993). Phosphoenolpyruvate: carbohydrate phosphotransferase systems of bacteria. Microbiol Rev 57, 543-594.

Shahidi, F., Arachchi, J. K. V. \& Jeon, Y. J. (1999). Food applications of chitin and chitosan. Trends Food Sci Technol 10, 37-51.

Suzuki, K., Suzuki, M., Taiyoji, M., Nikaidou, N. \& Watanabe, T. (1998). Chitin binding protein (CBP21) in the culture supernatant of Serratia marcescens 2170. Biosci Biotechnol Biochem 62, 128-135.

Suzuki, K., Uchiyama, T., Suzuki, M., Nikaidou, N., Regue, M. \& Watanabe, T. (2001). LysR-type transcriptional regulator ChiR is essential for production of all chitinases and a chitin-binding protein, CBP21, in Serratia marcescens 2170. Biosci Biotechnol Biochem 65, 338-347.

Suzuki, K., Sugawara, N., Suzuki, M., Uchiyama, T., Katouno, F., Nikaidou, N. \& Watanabe, T. (2002). Chitinases A, B, and C1 from Serratia marcescens 2170: enzymatic properties and synergism on chitin degradation. Biosci Biotechnol Biochem 66, 1075-1083.

Tews, I., Vincentelli, R. \& Vorgias, C. E. (1996). NAcetylglucosaminindase (chitobiase) from Serratia marcescens: gene sequence, and protein production and purification in Escherichia coli. Gene 170, 63-67.

Tsujibo, H., Miyamoto, J., Kondo, N., Miyamoto, K., Baba, N. \& Inamori, Y. (2000). Molecular cloning of the gene encoding an outermembrane-associated $\beta$-N-acetylglucosaminidase involved in chitin degradation system of Alteromonas sp. strain O-7. Biosci Biotechnol Biochem 64, 2512-2516.

Uchiyama, T., Kaneko, R., Yamaguchi, J., Inoue, A., Yanagida, T., Nikaidou, N., Regue, M. \& Watanabe, T. (2003). Uptake of $N, N^{\prime}-$ diacetylchitobiose [(GlcNAc)2] via the phosphotransferase system is essential for chitinase production by Serratia marcescens 2170 . J Bacteriol 185, 1776-1782. 
Vogler, A. P. \& Lengeler, J. W. (1989). Analysis of the nag regulon from Escherichia coli $\mathrm{K} 12$ and Klebsiella pneumoniae and of its regulation. Mol Gen Genet 219, 97-105.

Watanabe, T., Kimura, K., Sumiya, T., Nikaidou, N., Suzuki, K., Suzuki, M., Taiyoji, M., Ferrer, S. \& Regue, M. (1997). Genetic analysis of the chitinase system of Serratia marcescens 2170. J Bacteriol 179, 7111-7117.
Yang, C., Rodionov, D. A., Li, X., Laikova, O. N., Gelfand, M. S., Zagnitko, O. P., Romine, M. F., Obraztsova, A. Y., Nealson, K. H. \& Osterman, A. L. (2006). Comparative genomics and experimental characterization of $\mathrm{N}$-acetylglucosamine utilization pathway of Shewanella oneidensis. J Biol Chem 281, 29872-29885.

Edited by: H. L. Drake 